\title{
Nowy wskaźnik Parsevala mocy nieczynnej dwójnika elektrycznego
}

Streszczenie. Niniejszy artykuł jest kontynuacją badań nad nową definicją współczynnika mocy biernej dwójnika, który nie zależy od częstotliwości. W artykule przedstawiono nowe sformalizowane podejście do obliczania takiej miary mocy biernej w dziedzinie dyskretnej, za pomoca transformaty Z. Współczynnik mocy biernej nazwano wskaźnikiem Parsevala przez analogię do podobnego współczynnika stosowanego w analizie harmonicznej.

Abstract. This article is a continuation of the study on a new definition of reactive power factor of a two-terminal, which does not depend on frequency. The article presents a new formalized approach to calculate such a reactive-power measure in discrete time domain, with the use of Ztransform. The reactive power factor has been called Parseval's factor by analogy to a similar coefficient used in harmonic analysis. (New Parseval's reactive power factor of a two-terminal circuit).

Słowa kluczowe: operatory, filtry cyfrowe, moce bierna.

Keywords: operators, digital filters, reactive power.

\section{Definicje podstawowe}

Splot: działanie filtru cyfrowego (FC) zachodzi wg algorytmu splotowego:

$$
(A x)_{n}=\sum_{m=-\infty}^{\infty} A_{m} x_{n-m}=\left(\left(\sum_{m=-\infty}^{\infty} A_{m} z^{m}\right) x\right)_{n}
$$

$$
\begin{aligned}
& (A, B)=\sum_{n=-\infty}^{\infty} A_{n} B_{n}=\left(A_{n} B_{n}\right)(1)= \\
& =\frac{1}{2 \pi j} \oint_{|z|=1} A\left(z^{-1}\right) B(z) d(\ln z)
\end{aligned}
$$

gdzie: $\left\{A_{m}\right\}_{m=-\infty}^{\infty}$ - próbki odpowiedzi impulsowej filtru, z - operator opóźnienia jednostkowego

$$
(z X)_{n}=x_{n-1} \Rightarrow\left(z^{m} x\right)_{n}=x_{n-m}
$$

Filtr przyczynowy A:

8)

$$
A_{n}=0 \text { for } n<0
$$

Filtr stabilny:

$$
\sum_{n=-\infty}^{\infty}\left|A_{n}\right|<\infty \longleftrightarrow \forall_{z:|z| \leq 1}|A(z)|<\infty
$$

$$
A(z)=\sum_{m=-\infty}^{\infty} A_{m} z^{m}, z \in \mathbf{C}
$$

(2)

$$
A_{n}=\frac{1}{2 \pi j} \oint_{|z|=1} A(z) z^{-n} d(\ln z)
$$

Iloczyn skalarny sygnałów lub filtrów definiowany jest następująco:

$$
(A, B)=\sum_{n=-\infty}^{\infty} A_{n} B_{n}
$$

Rozkład biegunowy przyczynowego filtru $Y$ definiowany jest następująco $[4,5,6]$ :

$$
Y=H U
$$

gdzie: $\mathrm{H}$-filtr samosprzężony (hermitowski): $\mathrm{H}=\mathrm{H}^{\star}$

$U$ - filtr unitarny:

$$
U^{-1}=U^{*} \Rightarrow\|U\|^{2}=(U, U)=1
$$

Filtr unitarny ma reprezentację wykładniczą:

Filtrem sprzężonym do filtru A nazywa się filtr A*, który dla dowolnych sygnałów $\mathrm{x}$, y spełnia warunek:

$$
(A x, y)=\left(x, A^{*} y\right)
$$

Zachodzi: $A_{-n}=A_{n}$ w dziedzinie ' $n$ ', oraz $A^{*}(z)=A\left(z^{-1}\right)$ w dziedzinie ' $z$ '. W obu dziedzinach działają:

- twierdzenie Borela o splocie:

$$
\left(\sum_{m} A_{n-m} B_{m}\right)(z)=A(z) B(z)
$$

$$
\left(A_{n} B_{n}\right)(z)=\frac{1}{2 \pi j} \oint_{|w|=1} A\left(z w^{-1}\right) B(w) d(\ln w)
$$

- wzór Parsevala dla iloczynu skalarnego:

$$
U=e^{\phi}=\sum_{n=0}^{\infty} \frac{\phi^{n}}{n !}
$$

gdzie $\phi^{*}=-\phi$ filtr antyhermitowski Rozkład ortogonalny operatora unitarnego:.

$$
\begin{aligned}
& U=\frac{1}{2}\left(U+U^{*}\right)+\frac{1}{2}\left(U-U^{*}\right)= \\
& =\cosh (\phi)+\sinh (\phi)
\end{aligned}
$$

zachodzi:

$$
\begin{aligned}
& (\cosh (\phi), \sinh (\phi))=0, \\
& \|\cosh (\phi)\|^{2}+\|\sinh (\phi)\|^{2}=1, \\
& {[\cosh (\phi)]^{2}-[\sinh (\phi)]^{2}=\delta,}
\end{aligned}
$$

gdzie $\delta$-sygnał jednostkowy Kroneckera tj. 
$\delta_{n}= \begin{cases}1 & \text { for } n=0 \\ 0 & \text { for } n \neq 0\end{cases}$

Dla rozkładu biegunowego operatora przyczynowego $Y$ zachodzi:

$$
\left(Y, Y^{-1}\right)=\left(H U, H^{-1} U^{*}\right)=\left(U^{2}, 1\right)
$$

a także

$$
\begin{aligned}
& \|\cosh (\phi)\|^{2}=\frac{1}{4}\left(U+U^{*}, U+U^{*}\right)= \\
& =\frac{1}{2}\left[1+\left(U^{2}, 1\right)\right]=\frac{1}{2}\left[1+\left(Y, Y^{-1}\right)\right] \\
& \|\sinh (\phi)\|^{2}=\frac{1}{4}\left(U-U^{*}, U-U^{*}\right)= \\
& =\frac{1}{2}\left[1-\left(U^{2}, 1\right)\right]=\frac{1}{2}\left[1-\left(Y, Y^{-1}\right)\right]
\end{aligned}
$$

skąd wynika że:

$$
-1 \leq\left(Y, Y^{-1}\right) \leq 1
$$

a także, biorąc pod uwagę, że:

$$
\left(Y, Y^{-1}\right)=\sum_{n=0}^{\infty} Y_{n}\left(Y^{-1}\right)_{n}=1+\sum_{n=1}^{\infty} Y_{n}\left(Y^{-1}\right)_{n}
$$

otrzymuje się w ten sposób użyteczny wskaźnik mocy nieczynnej:

$$
\|\sinh (\phi)\|^{2}=-\frac{1}{2} \sum_{n=1}^{\infty} Y_{n}\left(Y^{-1}\right)_{n}
$$

lloczyn skalarnym $\left(Y, Y^{-1}\right) \quad$ jest pośrednim wskaźnikiem mocy nieczynnej. Przyjmuje on optymalną wartość $\left(Y, Y^{-1}\right)=1$ i wówczas $\|\sinh (\phi)\|^{2}=0$.

Dla $\left(Y, Y^{-1}\right)=-1$ dwójnik ma najgorsze wskaźniki energetyczne $\|\cosh (\phi)\|^{2}=0$ and $\|\sinh (\phi)\|^{2}=1$.

lloczyn skalarny $\left(Y, Y^{-1}\right)$ może być też wyznaczony w dziedzinie 'z', z użyciem wzoru Parsevala:

$$
\begin{aligned}
& \left(Y, Y^{-1}\right)=\frac{1}{2 \pi j} \oint_{|z|=1} Y(z) Y^{-1}\left(z^{-1}\right) d(\ln z)= \\
& =\frac{1}{2 \pi j} \oint_{|z|=1} Y\left(z^{-1}\right) Y^{-1}(z) d(\ln z)= \\
& =\frac{1}{2 \pi j} \oint_{|z|=1} \frac{1}{2}\left[\frac{Y(z)}{Y\left(z^{-1}\right)}+\frac{Y\left(z^{-1}\right)}{Y(z)}\right] d(\ln z)= \\
& =\frac{1}{2 \pi j} \oint_{|z|=1} \frac{1}{2}\left[\frac{Y^{2}+\left(Y^{*}\right)^{2}}{Y Y^{*}}\right] d(\ln z)
\end{aligned}
$$

gdzie: $\quad Y^{*}(z)=Y\left(z^{-1}\right)$.

W przypadku wymiernej, stabilnej zwarciowo i otwarciowo funkcji immitancji dwójnika $Y(z)$, bieguny funkcji podcałkowej cechuje symetria względem okręgu jednostkowego (rys. 1).

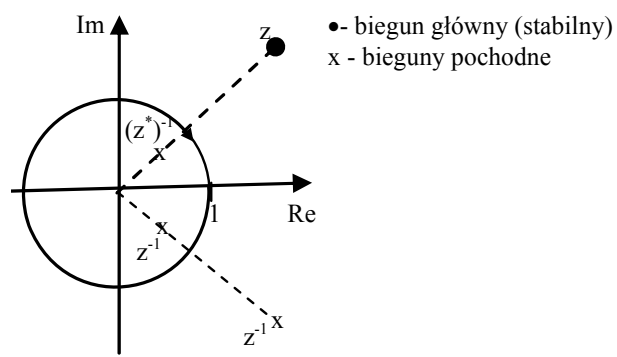

Rys.1. symetria biegunów względem okręgu jednostkowego

Kładąc pod całkę $Y=L / M$ gdzie $L(z)$ i $M(z)$ wielomiany od ' $z$ ', otrzymuje się też:

$$
\left(Y, Y^{-1}\right)=\frac{1}{2 \pi j} \oint_{|z|=1} \frac{1}{2}\left[\frac{\left(L M^{*}\right)^{2}+\left(L^{*} M\right)^{2}}{L L^{*} M M^{*}}\right] d(\ln z)
$$

Wewnątrz koła jednostkowego znajdują się bieguny funkcji $Y^{*}(z)$. Całkę okrężną zmiennej zespolonej można zastąpić całkę oznaczoną zmiennej rzeczywistej biorąc pod uwagę że

$$
\left.\frac{1}{2} \frac{Y^{2}+\left(Y^{*}\right)^{2}}{Y Y^{*}}\right|_{z=e^{j \omega}}=\left.\cos [2 \measuredangle Y(z)]\right|_{z=e^{j \omega}}
$$

Wówczas:

$$
\left(Y, Y^{-1}\right)=\left.\frac{1}{2 \pi} \int_{0}^{2 \pi} \cos [2 \measuredangle Y(z)]\right|_{z=e^{j \omega}} d \omega
$$

\section{Przykład1}

Nierówność $-1 \leq\left(Y, Y^{-1}\right) \leq 1$ gdzie

$\left(Y, Y^{-1}\right)=\left.\frac{1}{2 \pi} \int_{0}^{2 \pi} \cos [2 \measuredangle Y(z)]\right|_{z=e^{j \omega}} d \omega$

potwierdza wykres na rysunku

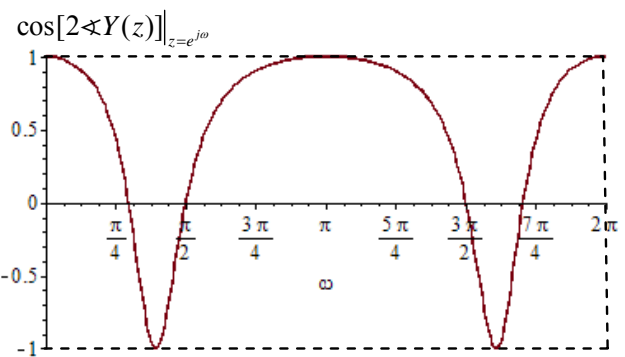

Rys. 2. Zakres zmienności $\left.\cos [2 \Varangle Y(z)]\right|_{z=e^{i \omega}}$ dla $Y(z)=\frac{a z^{2}+b z+c}{z^{2}+d z+e}$

Wykres funkcji zawiera się wewnątrz prostokąta $1 . .1,0 . .2 \pi$ dlatego pole powierzchni pod wykresem funkcji $2 \pi$ zawiera się pomiędzy polami prostokątów o powierzchniach $+1 \mathrm{i}-1$

\section{Przykład2}

Warto w szczególności rozpatrzeć dwa dwójniki elektryczne scharakteryzowane operatorem czystej konduktancji $Y_{1}(z)=a$ i reaktancji $Y_{2}(z)=a \ln (z)$, gdzie $\mathrm{a}=$ const wówczas: 


$$
\begin{aligned}
& \left(Y_{1}, Y_{1}^{-1}\right)=\frac{1}{2 \pi j} \oint_{|z|=1} d(\ln z)=\frac{1}{2 \pi j} \int_{0}^{2 \pi} d\left[\ln \left(e^{j \omega}\right)\right]=1, \\
& \left(Y_{2}, Y_{2}^{-1}\right)=\frac{1}{2 \pi j} \oint_{|z|=1} \frac{1}{2}\left[\frac{\ln (z)}{\ln \left(z^{-1}\right)}+\frac{\ln \left(z^{-1}\right)}{\ln (z)}\right] d(\ln z)= \\
& =\frac{1}{2 \pi j} \int_{0}^{2 \pi}(-1) d\left[\ln \left(e^{j \omega}\right)\right]=-1
\end{aligned}
$$

\section{Analiza w zbiorze liczb zespolonych}

Dla sygnałów harmonicznych prądu i napięcie sygnały i filtry scharakteryzowane są zwykłymi liczbami zespolonymi. lloczyn skalarny liczb zespolonych A i B określa wyrażenie

$$
(A, B)=\operatorname{Re}\left(A B^{*}\right)
$$

Rozkład biegunowy liczby zespolonej który naśladuje rozkład biegunowy filtru cyfrowego (lub na odwrót) ma postać:

$$
\begin{aligned}
& Y=H U, H^{*}=H
\end{aligned}
$$

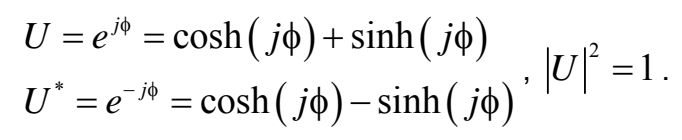

Tym razem

$$
\begin{aligned}
& \cosh (j \phi)=\frac{1}{2}\left(U+U^{*}\right)=\cos (\phi) \\
& \sinh (j \phi)=\frac{1}{2}\left(U-U^{*}\right)=j \sin (\phi)
\end{aligned}
$$

Rozkład $\cosh (j \phi)+\sinh (j \phi)$ jest ortogonalny, bo

$$
(\cosh (j \phi), \sinh (j \phi))=\operatorname{Re}\{\cos (\phi)[-j \sin (\phi)]\}=0
$$

a więc

$$
|\cosh (j \phi)|^{2}+|\sinh (j \phi)|^{2}=1
$$

Warto też zauważyć, że rolę normy $\|\bullet\| w$ dziedzinie filtrów w zbiorze liczb zespolonych przejmuje zwykły moduł.

Dalej otrzymuje się analogiczne relacje:

$$
\begin{gathered}
\left(Y, Y^{*}\right)=\left(H U, H^{-1} U^{*}\right)=\operatorname{Re}\left(U^{2}\right)=\cos (2 \phi) \\
|\cosh (j \phi)|^{2}=\frac{1}{4}\left(U+U^{*}, U+U^{*}\right)= \\
=\frac{1}{4} \operatorname{Re}\left[\left(U+U^{*}\right)\left(U+U^{*}\right)\right]= \\
=\frac{1}{2}\left[1+\operatorname{Re}\left(U^{2}\right)\right]=\frac{1}{2}\left[1+\left(Y, Y^{-1}\right)\right] \\
|\cosh (j \phi)|^{2}=\frac{1}{2}\left[1-\left(Y, Y^{-1}\right)\right]
\end{gathered}
$$

Zatem :

$$
\left(Y, Y^{-1}\right)=\operatorname{Re}\left(\frac{Y}{Y^{*}}\right)=\cos (2 \phi)
$$

Tym samym

$$
\begin{aligned}
& |\cosh (j \phi)|^{2}=\frac{1}{2}\left[1+\operatorname{Re}\left(\frac{Y}{Y^{*}}\right)\right]= \\
& =\frac{1}{2}[1+\cos (2 \phi)]=[\cos (\phi)]^{2} \\
& |\sinh (j \phi)|^{2}=\frac{1}{2}\left[1-\operatorname{Re}\left(\frac{Y}{Y^{*}}\right)\right]= \\
& =\frac{1}{2}[1-\cos (2 \phi)]=[\sin (\phi)]^{2}
\end{aligned}
$$

Wyniki te szczególności potwierdzają relację nierównościową $-1 \leq\left(\mathrm{Y}, \mathrm{Y}^{-1}\right) \leq 1$ otrzymaną $\mathrm{w}$ uogólnieniu na filtry cyfrowe.

$$
\begin{aligned}
& \left(Y, Y^{-1}\right)=\frac{1}{2 \pi j} \int_{-j \infty}^{j \infty} Y(s)[Y(-s)]^{-1} d s= \\
& =\frac{1}{2 \pi j} \int_{-j \infty}^{j \infty} \frac{1}{2}\left(\frac{Y}{Y^{*}}+\frac{Y^{*}}{Y}\right) d s= \\
& =\frac{1}{2 \pi j} \int_{-j \infty}^{j \infty} \frac{1}{2}\left[\frac{Y^{2}+\left(Y^{*}\right)^{2}}{Y^{*} Y}\right] d s
\end{aligned}
$$

gdzie: $Y^{*}(\mathrm{~s})=\mathrm{Y}(-\mathrm{s})$

Nie należy się spodziewać zbieżności całki osobliwej

$\left.\int_{-\infty}^{\infty} \cos [2 \measuredangle Y(s)]\right|_{s=j \omega} d \omega$

o czym łatwo się przekonać przyjmując model dwójnika elektrycznego scharakteryzowany operatorem czystej konduktancji $\mathrm{Y}(\mathrm{s})=\mathrm{a}=$ const:

$$
\left(Y, Y^{-1}\right)=\frac{1}{2 \pi} \int_{-\infty}^{\infty} d \omega \rightarrow \infty
$$

\section{Rozkład biegunowy filtrów periodycznych}

Operator periodyczny filtru SLS $\left\{A_{n}\right\}_{n=-\infty}^{\infty}$ ma postać następującą

$$
\widetilde{A_{n}}=\sum_{p=-\infty}^{\infty} A_{n+p N}, n \in\{0,1, \ldots, N-1\} \equiv \mathbf{N}
$$

operator sprzężony

$$
\begin{aligned}
& \widetilde{A_{n}^{*}}=\sum_{p=-\infty}^{\infty} A_{-n+p N} \stackrel{p \rightarrow p+1}{\longrightarrow} \\
& \longrightarrow \sum_{p=-\infty}^{\infty} A_{N-n+p N}=\widetilde{A_{N-n}}
\end{aligned}
$$

Operacją mnożenia w zbiorze filtrów periodycznych jest splot cykliczny 


$$
(A B)_{n}=\sum_{m \in \mathbf{N}} A_{n \ominus m} B_{m}
$$

gdzie: $n \Theta m$ - odejmowanie modulo $\mathrm{N}$ a iloczynem skalarnym jest

$$
(A, B)=\sum_{n \in \mathbf{N}} A_{n} B_{n}
$$

Operator przejścia filtru

$$
(A x)_{n}=\sum_{m \in \mathbf{N}} A_{m} x_{n \ominus m}=\left(\left(\sum_{m \in \mathbf{N}} A_{m} z^{m}\right) x\right)_{n}
$$

gdzie z -operator opóźnienia .

Wynika stąd, że $\mathrm{z}^{\mathrm{N}}=1$, bo $n \ominus N=n-N+N=n$ Jeżeli więc $z \in \mathbb{C}$ to:

$$
\left\{z: z^{N}=1\right\} \doteqdot \sqrt[N]{1}=\left\{w^{n}: n \in \mathbb{N}, w=e^{-j \frac{2 \pi}{N}}\right\}
$$

$\mathrm{W}$ operatorze przejścia filtru pojawia się tak zwana forma ' $z$ ' definiowana wielomianem:

$$
A(z)=\sum_{m \in \mathbf{N}} A_{m} z^{m}, z \in \sqrt[N]{1}
$$

Aby wprowadzić transmitancję odwrotną $A(z) \rightarrow A_{m}$ trzeba wyznaczyć

$$
\begin{aligned}
& \frac{1}{N} \sum_{z \in \mathbb{N} \sqrt{1}} A(z) z^{-n}=\frac{1}{N} \sum_{z \in \sqrt[N]{1}}\left(\sum_{m \in \mathbf{N}} A_{m} z^{m}\right) z^{-n}= \\
& =\sum_{m \in \mathbb{N}} A_{m}\left(\frac{1}{N} \sum_{z \in \mathbb{N} \sqrt{1}} z^{m-n}\right)
\end{aligned}
$$

Ponieważ dla wielomianu geometrycznego:

$$
\begin{aligned}
& \frac{1}{N} \sum_{z \in \mathbb{N} \sqrt{1}} z^{m-n}=\frac{1}{N} \sum_{p \in \mathbf{N}} w^{p(m-n)}= \\
& =\left\{\begin{array}{cl}
1 & \text { for } n-m=0 \\
\frac{1-w^{(m-n) N}}{1-w}=0 & \text { for } n-m \neq 0
\end{array}\right.
\end{aligned}
$$

stąd

$$
A_{n}=\frac{1}{N} \sum_{z \in \sqrt[N]{1}} A(z) z^{-n}, n \in \mathbf{N}
$$

Nietrudno otrzymać:

- twierdzenie Borela o splocie:

$$
\begin{gathered}
\left(\sum_{m \in \mathbb{N}} A_{n \ominus m} B_{m}\right)(z)=A(z) B(z), z \in \sqrt[N]{1} \\
\left(A_{n} B_{n}\right)(z)=\frac{1}{N} \sum_{w \in \sqrt[N]{1}} A\left(z w^{-1}\right) B(w)
\end{gathered}
$$

-wzór Parsevala

$$
\begin{aligned}
& (A, B)=\sum_{n \in \mathbb{N}} A_{n} B_{n}=\frac{1}{N} \sum_{z \in \mathbb{N} \sqrt{1}} A\left(z^{-1}\right) B(z)= \\
& =\frac{1}{N} \sum_{z \in \sqrt[N]{1}} A(z) B\left(z^{-1}\right)
\end{aligned}
$$

Zatem pośredni wskaźnik mocy nieczynnej może być obliczony za pomocą wzoru:

$$
\begin{aligned}
& \left(Y, Y^{-1}\right)=\sum_{n \in \mathbb{N}} \widetilde{Y}_{n}\left(\widetilde{Y}^{-1}\right)_{n}=\frac{1}{N} \sum_{z \in \mathbb{N} \sqrt{1}} \frac{Y(z)}{Y^{*}(z)}= \\
& =\frac{1}{N} \sum_{z \in \sqrt[N]{1}} \frac{1}{2}\left[\frac{Y(z)}{Y^{*}(z)}+\frac{Y^{*}(z)}{Y(z)}\right]= \\
& =\frac{1}{N} \sum_{z \in \mathbb{N} \sqrt{1}} \frac{1}{2}\left[\frac{Y^{2}+\left(Y^{*}\right)^{2}}{Y Y^{*}}\right](z)= \\
& =\frac{1}{N} \sum_{z \in \sqrt[N]{1}} \cos [2 \measuredangle Y(z)]
\end{aligned}
$$

\section{Wyznaczenie współczynnika mocy w dziedzinie częstotliwości}

Otrzymaną formułę (15) dla bezpośrednich wskaźników mocy czynnej i nieczynnej można rozszerzyć na dziedzinę widmową. Z wyrażeń :

$$
\begin{aligned}
& \|\sinh (\phi)\|^{2}=\frac{1}{2}\left[1-\left(Y, Y^{-1}\right)\right], \\
& \|\cosh (\phi)\|^{2}=\frac{1}{2}\left[1+\left(Y, Y^{-1}\right)\right],
\end{aligned}
$$

gdzie:

$$
\begin{aligned}
& \left(Y, Y^{-1}\right)=\sum_{n=0}^{\infty} Y_{n}\left(Y^{-1}\right)_{n}=1+\sum_{n=1}^{\infty} Y_{n}\left(Y^{-1}\right)_{n}= \\
& =\frac{1}{2 \pi j} \oint_{|z|=1} \frac{1}{2}\left(\frac{Y}{Y^{*}}+\frac{Y^{*}}{Y}\right)(z) d(\ln z)= \\
& =\frac{1}{2 \pi} \int_{-\pi}^{\pi} \cos (2 \measuredangle Y) d \omega
\end{aligned}
$$

wynikają następujące formuły:

$$
\begin{aligned}
& \|\sinh (\phi)\|^{2}=\frac{-1}{2 \pi j} \oint_{|z|=1} \frac{1}{4} \frac{\left(Y-Y^{*}\right)^{2}}{Y Y^{*}} d(\ln z)= \\
& =\frac{1}{2 \pi} \int_{-\pi}^{\pi}[\sin (\measuredangle Y)]^{2} d \omega \\
& \|\cosh (\phi)\|^{2}=\frac{1}{2 \pi j} \oint_{|z|=1} \frac{1}{4} \frac{\left(Y+Y^{*}\right)^{2}}{Y Y^{*}} d(\ln z)= \\
& =\frac{1}{2 \pi} \int_{-\pi}^{\pi}[\cos (\measuredangle Y)]^{2} d \omega
\end{aligned}
$$

oraz

$$
\begin{aligned}
& \left(Y, Y^{-1}\right)=\frac{1}{2 \pi} \int_{-\pi}^{\pi} \frac{Y_{1}^{2}-Y_{2}^{2}}{Y_{1}^{2}+Y_{2}^{2}} d \omega, \\
& \|\sinh (\phi)\|^{2}=\frac{1}{2 \pi} \int_{-\pi}^{\pi} \frac{Y_{2}^{2}}{Y_{1}^{2}+Y_{2}^{2}} d \omega, \\
& \|\cosh (\phi)\|^{2}=\frac{1}{2 \pi} \int_{-\pi}^{\pi} \frac{Y_{1}^{2}}{Y_{1}^{2}+Y_{2}^{2}} d \omega,
\end{aligned}
$$

gdzie (używając transformaty biliniowej): 


$$
\begin{aligned}
& Y(s) \rightarrow Y\left(j \frac{2}{\tau} \tan \frac{\omega}{2}\right)=Y_{1}(\omega)+j Y_{2}(\omega), \\
& \measuredangle Y(s) \rightarrow \measuredangle Y\left(j \frac{2}{\tau} \tan \frac{\omega}{2}\right)=\arctan \frac{Y_{2}(\omega)}{Y_{1}(\omega)} . \\
& \tau \text { - odstęp próbkowania }
\end{aligned}
$$

Współczynnik mocy $\left(Y, Y^{-1}\right)$ jest również uniwersalny w ten sposób, że operator admitancji może zostać zastąpiony przez operatora impedancji ( $Z Y=1)$.

\section{Przykład}

Przyjmując postać admitancji

$$
Y(s)=a+s \rightarrow Y=a+\left(j \frac{2}{\tau} \tan \frac{\omega}{2}\right)
$$

otrzymujemy:

$$
\Varangle Y=\operatorname{arctg}\left(\frac{2}{a \tau} \operatorname{tg} \frac{\omega}{2}\right) \underset{a \rightarrow 0}{\stackrel{a \rightarrow \infty}{\longrightarrow}} \pi / 2
$$

Z kolei przyjmując np. dwójnik RL, lub RC o admitancji

$$
Y(s)=\frac{\prod_{b}(b+s)}{\prod_{a}(a+s)}=\frac{L(s)}{M(s)},|\operatorname{deg} L-\operatorname{deg} M|=\{0,1\}
$$

gdzie a,b - rzeczywiste, otrzymujemy

$$
\begin{aligned}
& \Varangle Y=\sum_{b} \operatorname{arctg}\left(\frac{2}{b \tau} \operatorname{tg} \frac{\omega}{2}\right)-\sum_{a} \operatorname{arctg}\left(\frac{2}{a \tau} \operatorname{tg} \frac{\omega}{2}\right) \\
& \pm \operatorname{arctg}\left(\frac{2}{c \tau} \operatorname{tg} \frac{\omega}{2}\right) \underset{\omega \rightarrow \pi}{\stackrel{\omega \rightarrow-\pi}{\longrightarrow}-\pi / 2} \pi / 2 \text {; }
\end{aligned}
$$

dla a,b,c $>0$ (rys. 3)

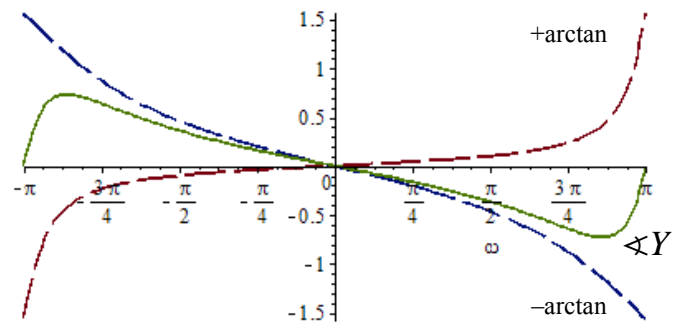

Rys. 3. Zmiany $\Varangle Y$ od $\omega$

oraz

$$
\|\sinh (\phi)\|^{2}=\frac{1}{2 \pi} \int_{0}^{2 \pi}[\sin (\measuredangle Y)]^{2} d \omega \in[0,1]
$$

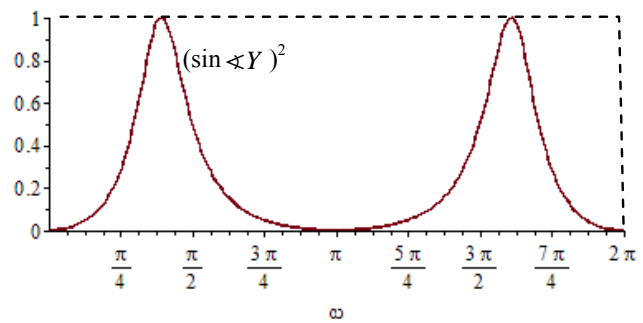

Rys. 4. Zakres zmienności $(\sin \Varangle Y)^{2}$ dla $Y(z)=\frac{a z^{2}+b z+c}{z^{2}+d z+e}$

\section{Wnioski}

Proponowany tu wskaźnik mocy nieczynnej dwójnika elektrycznego ma charakter obiektywny, to znaczy że nie zależy od rodzaju wymuszenia tylko od parametrów dwójnika. Jedynym parametrem subiektywnym może tu być odstęp próbkowania ale ma on niewielki wpływ na wartość wskaźnika mocy. Natomiast używane powszechnie wskaźniki (współczynniki) mocy to jest $\sin (\phi), \cos (\phi)$,

czy $\tan (\phi)$ są subiektywne bo funkcjonują jedynie dla wymuszeń sinusoidalnych o zadanej częstotliwości. Porównanie obiektywnych i subiektywnych wskaźników mocy przeprowadzono punkcie ' Analiza w zbiorze liczb zespolonych '.

Autorzy: prof. $d r$ hab. inż. Maciej Siwczyński, E-mail: e-3@pk.edu.pl; $d r$ inż. Marcin Jaraczewski, E-mail: jaracz@pk.edu.pl., Politechnika Krakowska, Wydział Inżynierii Elektrycznej i Komputerowej, Katedra Elektrotechniki i Elektroniki teoretycznej i Informatyki ul. Warszawska 24.

\section{LITERATURA}

[1] F. M. Atici, "A transform method in discrete fractional calculus", International Journal of Difference Equations 2, No. 2, pp. 165-176 (2007).

[2] Y. Lia, H. Shengb, Y. Q. Chen, "Analytical impulse response of a fractional second order filter and its impulse response invariant discretization", ELSEVIER Signal Processing 91, No. 3, pp. 498-507 (2011).

[3] T. Kaczorek, "Selected problems of fractional systems theory", Springer-Verlag, Berlin, 2012.

[4] M. Siwczyński, "The exponential and hyperbolic form of the periodical-convolution operator of signals in time domain and it's applications in power theory", Przegląd Elektrotechniczny 88, No. 6, pp. 194-197 (2012) [in Polish].

[5] M. Siwczyński, A. Drwal, S. Żaba, „The digital function filters algorithms and applications", Bulletin of the Polish Academy of Sciences. Technical Sciences 61, No. 2, pp. 371-377 (2013).

[6] M. Siwczyński, M. Jaraczewski, "New discrete reactive power factor definition of the two-terminal network", Bulletin of the Polish Academy of Sciences. Technical Sciences 65, No. 3, pp. 369-373 (2017). 